\title{
An affordable and automated imaging approach to acquire highly-resolved individual data - an example of copepod growth in response to multiple stressors
}

\author{
Jan Heuschele $^{\text {Corresp., } 1}$, Torben Lode ${ }^{1}$, Tom Andersen $^{1}$, Katrine Borgå $^{1}$, Josefin Titelman ${ }^{1}$ \\ ${ }^{1}$ Department of Biosciences, University of Oslo, Oslo, Norway \\ Corresponding Author: Jan Heuschele \\ Email address: janheu@ibv.uio.no
}

Individual trait variation is essential for populations to cope with multiple stressors and continuously changing environments. The immense number of possible stressor combinations and the influence of phenotypic variation makes experimental testing for effects on organisms challenging. The acquisition of such data requires many replicates and is notoriously laborious. It is further complicated when responses occur over short time periods. To overcome such challenges, we developed an automated imaging platform to acquire temporally highly resolved individual data. We tested this platform by exposing copepods to a combination of a biotic stressor (predator cues) and a toxicant (copper) and measured the growth response of individual copepods. We tested the automatically acquired data against published manually acquired data with much lower temporal resolution. We find the same general potentiating effects of predator cues on the adverse effects of copper, and the influence of an individual's clutch identity on its ability to resist stress, between the data obtained from low and high temporal resolution. However, when using the high temporal resolution, we also uncovered effects of clutch ID on the timing and duration of stage transitions, which highlights the importance of considering phenotypic variation in ecotoxicological testing. Phenotypic variation is usually not acknowledged in ecotoxicological testing. Our approach is scalable, affordable and adjustable to accommodate both aquatic and terrestrial organisms, and a wide range of visually detectable endpoints. We discuss future extensions that would further widen its applicability. 
1 An affordable and automated imaging approach to 2 acquire highly-resolved individual data - an example 3 of copepod growth in response to multiple stressors 4

5 Jan Heuschele, Torben Lode, Tom Andersen, Katrine Borgå, Josefin Titelman Department of Biosciences, University of Oslo, PO Box 1066, Blindern, 0316 Oslo, Norway

7 Corresponding Author:

8 Jan Heuschele

9 Department of Biosciences, University of Oslo, PO Box 1066, Blindern, 0316 Oslo, Norway

10 Email address: janheuschele@gmail.com

11

12

\section{Abstract}

Individual trait variation is essential for populations to cope with multiple stressors and continuously changing environments. The immense number of possible stressor combinations and the influence of phenotypic variation makes experimental testing for effects on organisms challenging. The acquisition of such data requires many replicates and is notoriously laborious. It is further complicated when responses occur over short time periods. To overcome such challenges, we developed an automated imaging platform to acquire temporally highly resolved individual data. We tested this platform by exposing copepods to a combination of a biotic stressor (predator cues) and a toxicant (copper) and measured the growth response of individual copepods. We tested the automatically acquired data against published manually acquired data with much lower temporal resolution. We find the same general potentiating effects of predator cues on the adverse effects of copper, and the influence of an individual's clutch identity on its ability to resist stress, between the data obtained from low and high temporal resolution. However, when using the high temporal resolution, we also uncovered effects of clutch ID on the timing and duration of stage transitions, which highlights the importance of considering phenotypic variation in ecotoxicological testing. Phenotypic variation is usually not acknowledged in ecotoxicological testing. Our approach is scalable, affordable and adjustable to accommodate both aquatic and terrestrial organisms, and a wide range of visually detectable endpoints. We discuss future extensions that would further widen its applicability.

\section{Introduction}


32 The life of any organism is a continuous struggle with different stressors, be it from other 33 organisms or the physical environment. Since the last century organisms are also exposed to

34 35 36 37 38 39 novel artificial substances of anthropogenic origin, such as chemical toxicants, as well as rapid changes in the environment due to human activities. In nature, stressors never act independently of one another. In addition to anthropogenic stressors, organisms must cope with natural biotic stress from parasites, competition for resources, and predation risk. While direct consumption is detrimental for prey, non-consumptive effects are complex (Heuschele et al. 2014), and include behavioral, morphological and physiological changes (Verity and Smetacek 1996). Most studies on mixture toxicity and multiple stressors focus either on interactive effects of two toxicants, or one pollutant in interaction with changes in the physical environment (Gunderson, Armstrong, and Stillman 2016). The effects of combined stressor exposures range from synergistic to antagonistic when compared to single stressor exposure (Rose et al. 2001, Fischer et al. 2012; Holmstrup et al. 2010), and the scales and timing of response differ widely, rendering it challenging to predict the outcome of additional stressors (e.g. Segner et al. 2014). However, such interactions between biotic stress and toxicants might be the rule rather than the exception and complicated indirect effects on predator-prey relationships seem to be common in aquatic communities (Rohr and Crumrine 2005, Langer-Jaesrich et al. 2010, Trekels et al. 2013).

Phenotypic differences within a population are another source of variation that complicate predicting multiple stressor effects. Recognizing this trait variation has profound and practical consequences for ecotoxicology, but also human medicine (Evans and Relling 2004).

Nevertheless, effects of stressors are often tested separately using laboratory populations of limited genetic diversity (OECD 2012, Macken, Lillicrap, and Langford 2015). The use of unique strains and clones inherently misses the ecotoxicological target "to predict effects in real populations" (Lam and Gray 2001).

The tremendous number of possible stressor combinations and the influence of phenotypic variation on the biota response poses a grand challenge for ecotoxicology, which is seemingly impossible to deal with, and yet unavoidable. Gathering individual data is more laborious compared to gathering pooled or group data, especially when it comes to following the development of individuals. The sensitivity of individuals is likely highest at specific life stages 
63 and stage transitions. Therefore, our focus should be to identify these states rather than only

64 addressing the larger time scale responses related to life span, fecundity and adult survival.

65 While the development rate of an animal can be estimated from daily observations, to actually

66 measure growth parameters e.g. length or examine rapidly occurring ontogenetic events e.g.

67 timing and duration of stage transitions, requires the frequent observer presence and potentially

68 the repeated handling of the experimental animals. Even the mere observer presence may

69 involuntarily alter the behavior and development of individuals (Mallet et al. 1987, Baker and

70 McGuffin 2007). Hence using traditional methods to gather individual data, such as manually

71 extracting, measuring, and placing them back into their holding container, can lead to biased

72 results from greater effects of handling than treatment.

73 This challenge calls for the application of efficient and automated testing platforms to execute

74 well-designed and manageable experiments that compare stress responses of organisms. In recent

75 years, automated experimental systems have been developed to monitor water quality by

76 quantifying motility and other endpoints in indicator species ranging from single celled Euglenas

77 (Tahedl and Häder 2001, Lee et al. 2012), over Daphnia (Häder and Erzinger 2017) to fish

78 (Cunha et al. 2008). There is a range of automated systems which find use as in real time

79 monitoring of behavioural responses of aquatic organisms (reviewed in Bae and Park 2014).

80 Among automated systems microfluidic Lab-on-a-chip systems (Campana and Wlodkowic 2018)

81 allow for the precise dosing in biochemical assays treatments. Due to their small size and cost

82 efficiency they could be used in high throughput screening of new chemicals. Measured

83 endpoints range from bioluminescence production (Zhao and Dong 2013), to viability

84 (Gammoudi et al. 2014), and motility (Huang et al. 2018). One drawback is however that small

85 volumes can limit the size of the testable animals (Campana and Wlodkowic 2018) and might

86 hinder a "natural" response to the treatment, especially when measured throughout the complete

87 development period of an organism. The aim of this study was thus to develop and test an

88 affordable automated imaging system that allows for the continuous observation of a large

89 number of separately kept individuals. We validated our approach by comparing our detailed

90 data and results to the ones from Lode et al. (2018) which are based on temporally less resolved

91 data from the same experiment.

92 We used copepods as model organisms, as they are key players in marine pelagic food webs and

93 the most abundant metazoans on the planet (Humes 1994; Naganuma 1996). In recent years, 
94 copepods are also increasingly used as models in ecotoxicology (Macken et al. 2015; Raisuddin 95 et al. 2007).

96

97 The experiment followed the development of individual copepods under the influence of a 98 biocide (copper) and a natural stressor in form of chemical cues of a fish predator. In our case 99 these include both kairomones, chemical cues emitted by the fish, which benefits the receiver 100 and potentially harms the emitter, and Schreckstoff, cues from eaten copepods that can warn the

101

102

103

104 105

106

107

108

109

110

111

112

113

114

115

116

117

118

119

120

121

122

123 other individuals. For simplicity we refer to these as predator cues in the remainder of the manuscript. We used a sublethal concentration of copper. We thus expected small but accumulative effects that would likely affect specific development stages. We anticipated that the highly resolved data would allow us to determine the most affected development stages, and also uncover subtle changes in growth trajectories and in the duration of stage transitions.

\section{Materials \& Methods}

\section{Automated platform for image acquisition}

We used an automatized imaging setup to follow individual growth at an hourly resolution, with a self-made experimental system that is capable of filming single culture plate wells repeatedly over the course of a copepod's development time. We used a DIY plotter kit (Makeblock Co., Ltd., XY-plotter Robot Kit V2, see Figure 1) as a basis for the system. On the movable platform, we mounted an upward facing infrared-capable camera with an image resolution of $2592 \times 1944$ pixels (Raspberry Pi NOIR with $\mathrm{C}$ mount and a $6 \mathrm{~mm}$ adjustable-focus lens). The images were saved as jpgs with a moderate compression of 85 to reduce file size while maintaining details, with 100 representing the maximum possible quality.

Above the camera system, we installed a platform made of transparent Plexiglas. On this, we placed four 24-well plates containing the animals. Two stepper motors move the camera position. Instructions are taken from a python script on a microcomputer (Raspberry Pi), which also controls the camera. We programmed the system to sequentially take one image $(2592 \times$ 1944 pixels) of each well approximately every hour, for a period of 13 days. Two LED lamps (Camlink CL-Studio10) provided constant illumination to the setup from opposing sides, providing dark field imaging conditions. After each well plate the system reset its positioning 
124 system using two contact switches at each axis. This prevents a continuous systematic error in 125 cases when the system got misaligned. We later discovered that such errors occurred sporadically 126 to be due to non-optimal baud rate settings of the serial port. The source code for the imaging 127 system is included in the as supplemental file $\mathrm{S} 1$. The material costs of the system summed up to 128 less than 500 EUROs.

130 The build of the setup is for the largest part easy as the plotter kit is targeted at juveniles. The 131 execution of the script and adding changes to the number of plates, timing of recordings can be 132 done by any person with basic python knowledge. User-friendliness could however easily be 133 improved by adding a simple graphical user interface to the script.

\section{Experiment and animals}

136 We tested the platform with an experiment on combined effects of predator cues and copper 137 exposure on copepod age and size at maturity.

138 We used the harpacticoid copepod Tigriopus brevicornis as the model organism. The laboratory 139 stock cultures originated from a splash water pool in Drøbak (Norway) and one from Tjärnö

140 (Sweden). We kept the stock cultures at $30 \mathrm{psu}, 18^{\circ} \mathrm{C}$ and a 12/12 light dark cycle for more than 141 six months before the experiment. Stock cultures were fed ad libitum three times a week an equal 142 mix of Rhodomonas salina, Isocrysis galbana, and Dunaniella tertiolecta.

143 Prior to the experiment, we picked single females with egg sacs from the stock culture. We 144 placed them individually into 24 well plates. Every 30 minutes we checked manually if nauplii 145 had hatched. If nauplii were detected, we registered the time, assigned a clutch ID and removed 146 the female. The nauplii were then placed individually in wells containing $2.5 \mathrm{ml}$ of water with 147 the respective treatment.

148 We exposed copepods to one of four treatments: predation risk (predator cues), copper (20 $\mu \mathrm{g} \mathrm{L}^{-}$

$149^{1}$ ), combined predation risk and copper $\left(20 \mu \mathrm{g} \mathrm{L}^{-1}\right)$, or control (pure seawater). All seawater was 150 taken from the outer Oslofjord and filtered at $1.2 \mu \mathrm{m}$ prior to use. We prepared seawater with 151 predator cues by incubating three-spined stickleback (Gasterosteus aculeatus) for $48 \mathrm{~h}$ in filtered 152 seawater at $18^{\circ} \mathrm{C}, 30 \mathrm{psu}, 2$ fish $\mathrm{1}^{-1}$. The fish were fed with T. brevicornis first at initiation, and 153 once more after $24 \mathrm{~h}$. Following incubation and removal of fish, the water was filtered (GF/C, $1541.2 \mu \mathrm{m})$ and frozen $\left(-18^{\circ} \mathrm{C}\right)$. Water without predator cues was prepared similarly but without 
155 addition of fish and copepods. These frozen bottles were thawed daily to prepare the exposure 156 solutions. For $\mathrm{Cu}$ and the combined treatment, we then added $\mathrm{Cu}$ through a 2-step dilution 157 process of a $0.1 \mathrm{M} \mathrm{CuSO} 4$ stock solution. Instead of $\mathrm{Cu}$ we similarly added distilled water for 158 the control and the predation risk treatment.

159 We replenished $72 \%$ of the exposure solution daily for each individual. We inspected the 160 individuals daily using a binocular microscope and recorded survival, took a photo for 161 subsequent length measurements, and most importantly assessed the development stage. We 162 used the numbers of exuviae to determine the stage of the copepods. For a more detailed 163 description of treatment preparation and the general procedure see Lode et al. (2018).

164

165

166

167

168

169

170

171

172

173

174

175

176

177

178

179

180

181

182

183

184

185

\section{Image acquisition}

We incubated 72 individual copepods from 9 clutches of different mothers. One was lost during the setup of the experiment, and two could not be followed due to a misalignment of the robot. From the 69 other ones, we managed to extract on average 168.9 \pm 31.1 measurements for each individual during development. The upper number of images and thus the maximum amount of measurements was 438 .

\section{Length measurements}

In total, we acquired 11657 images that were suitable for measurements. Some of the other pictures were misaligned due to occasional glitches in the computer drives platform, or because the system took them during the daily water change, when the wells where removed from the platform (Lode et al. 2018) These images had to be removed from the analysis. We imported the images into the imaging software Fiji (Schindelin et al. 2012). The high magnification reduced the depth of field and the copepods could only be measured when they were in close proximity of the bottom (Figure $1 \mathrm{C}$ ). If the animal was distinctly visible and in focus, we manually measured its length. Due to the benthic lifestyle of the nauplii compared to the demersal behavior of adults, we were able to obtain more measurements from younger stages. To compare the length measurement from machine images and the one obtained from microscope image by Lode et al. (2018), we calculated a daily average of the machine length measurements.

\section{Moult from nauplii to copepodite}


186 We determined the time of moult to the first copepodite stage (i.e. moment) by manually 187 screening the pictures for the first occurrence of the copepodite stage. Earlier moults from 188 nauplii to nauplii or moults from copepodite to copepodite were not as obvious and could not be 189 easily determined directly from the images. Sometimes a water change, a temporary 190 misalignment of the camera, or the temporary removal during the manual screening led to 191 unusable pictures. If such unusable pictures preceded the first appearance of a copepodite stage, 192 we were not able to accurately determine the time-point. To be able to account for the varying 193 degrees of uncertainty we noted down the number of "uncertain" pictures, and included this 194 information in the statistical analysis.

195 From the images we saw that most nauplii remained motionless for several frames during their 196 transition to the first copepodite stage. To be able to determine whether the treatments influenced 197 the moult duration to copepodite, we recorded the number of "motion free" pictures before the 198 first appearance of the copepodite. We included the first picture in which they were detected in 199 the same position.

200

201 Statistical analysis

202 All statistical analyses and data manipulation were conducted using the statistical software $\mathrm{R}$ 203 (Version 3.5.0) (R Core Team 2018). We used all acquired length measurements for the 204 analyses. We converted the measurement time to the actual age of the individual using the time 205 of birth ( \pm 30 minutes uncertainty). To meet normality assumptions, copepodite transition time 206 and length data were log-transformed before analysis, and data of the duration of the nauplii to 207 copepodite transition was square root transformed. If not otherwise stated, we used an 208 information theoretic approach to select the best model for the linear models based on the 209 corrected Akaike information criterion (AICc) for all measured response variables. If there were 210 several models within a $\triangle \mathrm{AICc}<2$ of the best one, we averaged model estimates. We used the 211 package MuMIn (Bartoń 2018) for model selection and averaging.

212 To test whether length measurements from the images where comparable to the manual 213 measurements from Lode et al. (2018), we used a linear model with machine as dependent, 214 microscope measurements as fixed factor. and individual ID as random factor to control for 215 repeated measurements of the same individual. We also included treatment as fixed factor in the 216 initial model, to test for differences in measurement "accuracy" between treatments. 
217 We tested the influence of treatment and clutch ID on transition timing from the last nauplii to 218 the first copepodite stage with a two-way ANOVA. We allowed for an interaction between both 219 independent factors in the initial model. We controlled for the uncertainty in transition time, by 220 including the number of "uncertain frames" as weights in the analysis.

221 To test whether males and females were affected differently by the treatments, we censored the 222 data to include only matured individuals. We then examined the influence of treatment and 223 224 gender in a separate model with these two factors as fixed factors, and copepodite transition time as the dependent variable. As before we allowed for all interactions between the independent 225 factors and the number of uncertain frames as the weighting factor. We tested for differences between clutch IDs and treatment in the moult duration of the nauplii to copepodite transition using a linear model with duration as dependent variable, and clutch ID and treatment as fixed factors. We allowed for interactions between the fixed factors in the initial model, and always included the number of uncertain frames as the weighting factor. We tested for the influence of copper, predator cue, and clutch ID on length development using general additive models (GAM) with thin plate regression splines, using the mgcv-package (Version 1.8 - 23) for general additive modelling (Wood 2011, Wood 2003). Our models included individual ID as random factor, allowing for a random smoothing over time for each individual. In this case, to choose the best model, we started out with a model allowing for full interactions between the fixed factors and took advantage of the inbuilt model selection tool of the mgcv-package where the smoothing parameter estimation allows for model terms to approach zero. This procedure results in a final best fitting model. The final model parameters and smoothing functions were then evaluated using the function gam.check () and based on k', estimated degrees of freedom (edf) and p-values. We further visually inspected the distribution of the residuals, quantile-quantile plots, and residual vs linear predictions.

241 Except for the moult from nauplii to copepodite, it was challenging to determine the exact time 242 point from one nauplii stage to the next by manual (visual) inspection of the acquired images. 243 Therefore, we tested for differences in transition timing between nauplii stages by analysing the 244 predicted individual growth increments over time. For this, we first ran a GAM that included 245 random smoothers for each individual only. We then calculated isochronal body length 246 predictions on a 72-minute resolution, similar to the one of the raw data. To not use the model 247 beyond the data range, individual level length predictions were limited to the respective time the 
248 individuals spent in the experiment. From these, we derived the individual growth increments, 249 which showed distinct peaks representing the stage transition phases.

250 We then used a new GAM with growth increments as the dependent factor to test for interactive 251 effects of Clutch ID, copper and predator cues on the transition dynamics. We also included 252 negative predictions of growth to keep the normally distributed nature of the data and to 253 emphasize the growth spurts during moults, although they are biologically impossible in this 254 species.

255 To test whether copper, predator cues, or clutch ID influenced the final size of the copepods at 256 the end of the experiment we restricted the data to measurements taken during the last day of the 257 experiment and averaged them for each individual. We then analyzed this relationship using a 258 linear model with copper, predator cues, and clutch ID as independent factors, and the log259 transformed (averaged) body length as the dependent factor. In this case the endpoint size 260 encompasses both potential effects of developmental delay and on size. To test whether 261 treatment influenced only the final size of males and females differently we further restricted the 262 averaged length data to include only lengths of fully matured individuals. We then used a similar 263 model structure to the previous one but added gender as a fixed factor in the initial model.

\section{Results}

265 The daily manually-measured length data by Lode et al. (2018) and data acquired using the robot 266 table correlated well (estimate $=0.90, \mathrm{SE}=0.01, \mathrm{~F}_{1,524}=4515, \mathrm{P}<0.001$ ), although automated 267 length estimates were generally larger than the manual measurements, especially during the early 268 copepodite stages (Figure 2). Treatments did not influence the relationship between machine and 269 manual measurements.

270 Copper delayed the time of the moult from nauplii $\mathrm{N} 6$ to copepodite $\mathrm{C} 1$, while the transition was 271 independent of an individual's clutch ID (Figure 3, Table 1). Model estimates for the N6 to C1 272 transition timing from nauplii to copepodite in males and females were similar, and the effect 273 was driven by individuals that did not reach maturity by the end of the experiment (Figure 3).

274 In contrast to the time of moult, the moult duration from nauplii N6 to copepodite $\mathrm{C} 1$ was mainly 275 influenced by the individual's clutch ID in interaction with copper (Figure 4, Table 1).

276 Individuals of six clutches showed a reduced moult duration, while in the other clutches the 
277 duration was prolonged compared the control (Figure 4). The duration of the moult ranges from

278 the time between two subsequent recordings ( $\sim 72 \mathrm{~min})$ to more than 400 minutes.

279 The development of individuals, measured as individual growth, is influenced by a complex

280 interactive effect of copper, predator cues and clutch ID (Figure 5, Figure S1, Table 2). The

281 treatments left two clutches unaffected (Figure S1), while individuals in all other families

282 responded with delayed growth. When we focus on the treatment effects, copper alone delayed

283 development while predator cues did not have an impact. The combination of both led to a

284 stronger delay in the late copepodite stages (Figure 5).

285 The conversion of length measurements to growth increments showed distinct peaks which

286 revealed the moults of the copepods, with variation in growth increments explained by an

287 interaction between Clutch ID, copper and predator cues (Figure 6, Table 3). Overall, the most

288 significant delay in development occurs in individuals exposed to copper and predator cues

289 combined when they metamorphose from nauplii to first copepodite (Figure 6), which confirms

290 the results of the manually screened timing of this major moult (Figure 3; Lode et al. 2018).

291 However already during the third transition the averaged peak height is reduced in the combined

292 stressor treatment, which indicates a larger variability between exposed individuals. With an

293 increased age of the individuals at the specific developmental stages, the moult cycles became

294 less synchronized and measurement error became larger, and an overall trend between treatments

295 was harder to detect, but also detecting individual peaks or moults becomes harder (see Figure

296 6). Therefore, we refrained from analyzing intermoult durations based on detected growth

297 maxima. However, the peak heights of the averaged growth increments are highest in the control

298 and predator cues treatment compared to the other two treatments, which means that there was

299 less variation in transition timing in control and predator cues treatment. Or in other words, the

300 effects of copper seem to be strongly affected by individual and clutch variation.

301 The final body size at the end of the experiment depends on additive effects of treatment and

302 Clutch ID. Both competing models $(\triangle \mathrm{AICc}<2)$ included additive effects by copper and clutch

303 ID, while only one had a negative effect of predator cues (Table 4). Some clutches were

304 unaffected by treatment while for most others the exposure by both treatments led to reduced

305 length (Figure S1). However, the 23 unmatured copepodites at the end of the experiment in the

306 copper and combined treatment biased these results. Therefore, we restricted the analyses to

307 include mature individuals only $(n=49)$. The analysis showed several competing models (Table 
308 4), which revealed that sex is the most important factor and to a lesser degree Clutch ID and

309 copper. In general, males were slightly larger than females (Table 4).

310

\section{Discussion}

312 The intensity of adverse responses to toxicants in the natural environment is challenging to

313 predict due to the almost infinite number of possible interactions with biotic and abiotic factors

314 (Segner et al 2014), and begs for efficient methods to handle many replicates. In this study, we

315 used an automated imaging approach to measure the combined impact of a toxicant (copper) and

316 a biotic stressor (predator cues) on copepod development. We validated and evaluated the added

317 benefits of our approach with the findings obtained using traditional manual methods at a much

318 lower time resolution (see Lode et al. 2018). We find the same complex interactions between the

319 copper and predator cues treatment, and individual's clutch ID, in determining the growth

320 trajectory of an individual facing multiple stressors.

321

322 Compared to the daily measurements of Lode et al. (2018) our highly-resolved data allowed us to 323 zoom in on individual moult events, which is not possible for large sample sizes using traditional 324 methods. Especially during the naupliar stages, we detected clear peaks of moult events. These 325 revealed that the treatment effects first affected the N3 transition and got more pronounced from 326 then onwards. The biggest effect is visible during the naupliar to copepodite (N6-C1)

327 metamorphosis. The significant differences between individuals and different clutches in their 328 response to the treatments led to a wider distribution of the moult timings (Figure 6). The strong 329 influence of an individual's clutch ID on the major intermoult duration also suggests a genetic 330 role in the resistance to multiple stressors, which is a major challenge in ecotoxicology (Evenden 331 and Depledge 1997, Wirgin and Waldman 2004).

332

333 The concurrent results show the potential of our semi-automated system to tackle large sample 334 sizes and detect small developmental differences in individual organisms, while still reducing the 335 workload and the handling of the animals. Our setup can thus ease the collection of individual 336 trait data and be used to answer questions in both toxicology and ecology. A focus on trait-based responses is especially helpful in studying the responses to multiple stressors. While we used it 
338 for small aquatic invertebrates, the imaging system is customizable and adjustable to

339 accommodate different container- and species sizes. It thus is in line with the successful use of

340 automatic monitoring systems in ecotoxicology like for example the Multispecies Freshwater

341 BiomonitorTM (Gerhardt and Schmidt 2002), LeDaphNet (Rutter et al. 2016), and DaphniaTox

342 (Häder et al. 2017) and lab-on-a-chip systems (Zhu et al. 2015, Campana and Wlodkovic 2018)

343 In its current state, the system can reliably capture the size and movement of animals with a

344 primarily benthic lifestyle. Examples include surface cruising animals such as benthic copepods,

345 snails, trematodes and nematodes. Using a bottom mounted camera works best when individuals

346 are close to the bottom of the holding container, or in our case the well plate. Potential research

347 questions include testing the influence of abiotic and biotic factors on the settlement of

348 planktonic larvae of benthic animals, growth development of invertebrates at different nutrient

349 concentrations, egg hatching times, and in this context also the factors which drive the

350 emergence of resting eggs. Our system can even quantify the behavioral variability in

351 populations from recorded movie sequences. As individual variability is the foundation and

352 currency of personality research, i.e. the study focusing on repeatable and correlated behaviors,

353 the imaging system can easily be used to capture consistent differences between individuals and

354 in consequence "personalities" of animals. In recent years it has become clear that such

355 characteristics that are not only present in "higher" organisms but also in invertebrates (Kralj-

356 Fiser and Schuett 2014; Sih, Bell, and Johnson 2004).

357 The depth of field of the camera is one of the apparent drawbacks of the current system. With

358 increasing age, the copepods became increasingly active and explored the whole water column.

359 Thus, the number of images from which we could reliably determine body length decreased with

360 age. This problem could be solved by using a smaller aperture or by adding a servomotor to the

361 camera. Moving the plane of focus while recording a movie, would increase the chances of

362 acquiring a sharp and complete image of the animal.

363 While the recording of the animals is automatized, our approach currently still relies on the

364 manual screening and measuring of the images. This step is necessary due to the imperfect image

365 quality (variable light conditions, occasional blurriness). An even background illumination using

366 electroluminescent sheets or diffuse LED light sources does however increase the image quality

367 to a point where it would be possible to implement image analyses based on neural networks. For 368 example, using the tensorflow library (Abadi et al. 2016) we could then automatically classify 
369 stage data, measure size data and other traits (gut content). The type of images could also be

370 analyzed using crowd-based annotation services such as Quanti.us (Hughes et al. 2018).

371

372 Conclusions

373 Our results illustrate the need to study the interactive effects of natural and anthropogenic

374 stressors, and they underscore the necessity to consider the phenotypic and genetic variation in

375 stress response if we want to use ecotoxicological studies to predict the consequences of

376 toxicants for natural populations. Our system takes the idea of autosamplers, lab-on-a chip and

377 other high-throughput ideas, and applies it to questions related to the development and

378 potentially the behavior of small invertebrates. It uncovered differences in moult duration and the

379 timing of copepod metamorphosis which would be difficult to detect using manual approaches.

380 Given that it is easy to build, affordable and runs with open source imaging and analysis

381 software, it can be scaled to accommodate for high-throughput testing of multiple treatment

382 combinations and gradients. When data are gained at both individual and population levels, they

383 can be combined conceptually in adverse outcome pathways and increase the value of risk

384 assessment in ecotoxicology (Kramer et al. 2011).

385

386

387

388

389

390

391

392

393

394

395

396

397

398

\section{Acknowledgements}

We thank Rita Amundsen for algae culture maintenance, the workshop at IBV for help with building the experimental setup, and the UiO Aquaria facilities, in particular Haaken Hveding Christensen, for keeping the sticklebacks, . This is a contribution to the prioritized research group LUMS (Life history under multiple stressors), financed by the Department of Biosciences, UIO.

\section{References}

Abadi, M. et al. 2016. "TensorFlow: A System for Large-Scale Machine Learning TensorFlow: A System for Large-Scale Machine Learning." 12th USENIX Symposium on Operating Systems Design and Implementation (OSDI'16) 265-284.

Bae, M. J. and Y. S. Park. 2014. Biological early warning system based on the responses of aquatic organisms to disturbances: a review. Science of the Total Environment, 466, 635649. 
399

400

401

402

403

404

405

406

407

408

409

410

411

412

413

414

415

416

417

418

419

420

421

422

423

424

425

426

427

428

429

Baker, R. L. and M. McGuffin. 2007. "Technique and Observer Presence Affect Reporting of Behavior of Damselfly Larvae." Journal of the North American Benthological Society 26:145-151.

Bartoń, K. 2018. “MuMIn: Multi-Model Inference.” Retrieved (https://cran.rproject.org/package=MuMIn).

Campana, O. and D. Wlodkowic. 2018. "Ecotoxicology goes on a chip: embracing miniaturized bioanalysis in aquatic risk assessment." Environmental science \& technology, 52(3): 932946.

Cunha, S. R., Gonçalves, R., Silva, S. R. and Correia, A. D. 2008. “An automated marine biomonitoring system for assessing water quality in real-time." Ecotoxicology 17: 558-564.

Evans, W. E. and M. V. Relling. 2004. "Moving towards Individualized Medicine with Pharmacogenomics." Nature 429: 464-468.

Evenden, A. J. and M. H. Depledge. 1997. "Genetic susceptibility in ecosystems: The challenge for ecotoxicology." Environmental health perspectives 105: 849-854.

Fischer, B., Roffler S. and Rik I. L. Eggen. 2012. "Multiple Stressor Effects of Predation by Rotifers and Herbicide Pollution on Different Chlamydomonas Strains and Potential Impacts on Population Dynamics.” Environmental Toxicology and Chemistry 31: 28322840 .

Gammoudi, I. et al. 2014. "Enhanced bio-inspired micro- sensor based on microfluidic/bacterial/love wave hybrid structure for continuous control of heavy metals toxicity in liquid medium. Sens. Actuators, B, 198: 278-284.

Gerhardt, A. and S. Schmidt. 2002. "The Multispecies Freshwater Biomonitor a Potential New Tool for Sediment Biotests and Biomonitoring." Journal of Soils and Sediments 2: 67-70.

Gunderson, Alex R., Eric J. Armstrong and Jonathon H. Stillman. 2016. "Multiple Stressors in a Changing World: The Need for an Improved Perspective on Physiological Responses to the Dynamic Marine Environment.” Annual Review of Marine Science 8: 357-378.

Häder, Donat-P. and Gilmar S. Erzinger. 2017. "Daphniatox - Online Monitoring of Aquatic Pollution and Toxic Substances." Chemosphere 167:228-235.

Heuschele, J. et al. 2014. "Non-Consumptive Effects of Predator Presence on Copepod Reproduction: Insights from a Mesocosm Experiment.” Marine Biology 161: 1653-1666. Holmstrup, M. et al. 2010. "Interactions between Effects of Environmental Chemicals and 
430

431

432

433

434

435

436

437

438

439

440

441

442

443

444

445

446

447

448

449

450

451

452

453

454

455

456

457

458

459

460

Natural Stressors: A Review." Science of the Total Environment 408: 3746-3762.

Huang, Y., Campana, O. and Wlodkowic, D. 2017. "A millifluidic system for analysis of daphnia magna locomotory responses to water-born toxicants." Scientific reports, 7: 17603.

Hughes, A. J. et al. 2018. "Quanti. us: a tool for rapid, flexible, crowd-based annotation of images." Nature methods, 15: 587-590.

Humes, A. G. 1994. “How Many Copepods ?” Hydrobiologia 292/293:1-7.

Kralj-Fiser, S. and W. Schuett. 2014. "Studying Personality Variation in Invertebrates: Why Bother?" Animal Behaviour 91:41-52.

Lam, P. K. S. and J. S. Gray. 2001. "Predicting Effects of Toxic Chemicals in the Marine Environment." Marine Pollution Bulletin 42: 169-73.

Langer-Jaesrich, M., Kienle, C., Köhler, H.R. and A. Gerhardt. 2010. "Impairment of Trophic Interactions between Zebrafish (Danio rerio) and Midge Larvae (Chironomus riparius) by Chlorpyrifos." Ecotoxicology 19: 1294-1301.

Lee, S. A., Zheng,G. Mukherjee, N. and C. Yang. 2012. "On-Chip Continuous Monitoring of Motile Microorganisms on an EPetri Platform.” Lab on a Chip 12: 2385.

Lode, T. et al. 2018. "Predation risk potentiates toxicity of a common metal contaminant in a coastal copepod" Environmental Science \& Technology 52: 13535-13542.

Macken, A., Lillicrap A. and K. Langford. 2015. "Benzoylurea Pesticides Used as Veterinary Medicines in Aquaculture: Risks and Developmental Effects on Nontarget Crustaceans." Environmental Toxicology and Chemistry 34:1533-1542.

Mallet, J. et al. 1987. "Handling Effects in Heliconius : Where Do All the Butterflies Go ?" Journal of Animal Ecology 56: 377-386.

Organisation for Economic Co-operation and Development. 2012. Test No. 211: Daphnia magna reproduction test. OECD Publishing.

Naganuma, T. 1996. “Calanoid Copepods: Linking Lower-Higher Trophic Levels by Linking Lower-Higher Reynolds Numbers.” Marine Ecology Progress Series 136:311-313.

R Core Team. 2018. "R: A Language and Environment for Statistical Computing.” Retrieved (https://www.r-project.org/).

Raisuddin, S., Kwok, K. W. H, Leung, K. M. Y., Schlenk, D. and J. S. Lee. 2007. “The Copepod Tigriopus: A Promising Marine Model Organism for Ecotoxicology and Environmental Genomics." Aquatic Toxicology 83: 161-173. 
461 Rohr, J. R. and P. W. Crumrine. 2005. "Effects of an herbicide and an insecticide on pond 462 community structure and processes.” Ecological Applications 15: 1135-1147.

463 Rose, R. M., Warne, M. S. J. and R. P. Lim. 2001. "The presence of chemicals exuded by fish 464 affects the life-history response of Ceriodaphnia $c f$. dubia to chemicals with different 465 mechanisms of action.” Environmental toxicology and chemistry, 20: 2892-2898. 466 Rutter, E. M. et al. 2017. “LeDaphNet: An Automated Assessment of Daphnia Magna 467 Populations Using Digital Imaging and Machine Learning," North Carolina State 468 University. Center for Research in Scientific Computation.

469 Schindelin, J. et al. 2012. Fiji: an open-source platform for biological-image analysis. Nature $470 \quad$ methods, 9.7: 676.

471 Segner, H. M., Schmitt-Jansen, M. and S. Sabater. 2014. Assessing the impact of multiple 472 stressors on aquatic biota: the receptor's side matters. Environmental Science and 473 Technology, 48: 7690-7696.

474 Sih, A., Bell, A. and J. C. Johnson. 2004. "Behavioral Syndromes: An Ecological and 475 Evolutionary Overview." Trends in ecology \& evolution 19:372-378.

476 Tahedl, H. and D. P. Häder. 2001. "Automated biomonitoring using real time movement analysis 477 of Euglena gracilis.” Ecotoxicology and Environmental Safety, 48: 161-169.

478 Trekels, H., Van de Meutter, F. and R. Stoks. 2013. "Predator Cues Magnify Effects of the 479 Pesticide Endosulfan in Water Bugs in a Multi-Species Test in Outdoor Containers.” 480 Aquatic Toxicology 138, 116-122.

481 Verity, P. G. and V. Smetacek. 1996. “Organism Life Cycles, Predation, and the Structure of 482 Marine Pelagic Ecosystems.” Marine Ecology Progress Series 130: 277-293.

483 Wirgin, I. and J. R. Waldman. 2004. "Resistance to contaminants in North American fish 484 populations." Mutation Research/Fundamental and Molecular Mechanisms of Mutagenesis 485 552: 73-100.

486 Wood, S. N. 2003. “Thin-Plate Regression Splines.” Journal of the Royal Statistical Society (B) $487 \quad 65: 95-114$.

488 Wood, S. N. 2011. "Fast Stable Restricted Maximum Likelihood and Marginal Likelihood 489 Estimation of Semiparametric Generalized Linear Models." Journal of the Royal Statistical $490 \quad$ Society (B) $73: 3-36$.

491 Zhao, X. Y. and Dong, T. 2013. “A Microfluidic Device for Continuous Sensing of Systemic 
492 Acute Toxicants in Drinking Water.” Int. J. Environ. Res. Public Health, 10: 6748-6763.

493 Zhu, F. et al. (2015). Automated lab-on-a-chip technology for fish embryo toxicity tests

494 performed under continuous microperfusion $(\mu \mathrm{FET})$. Environmental science \& technology, 495 49: 14570-14578.

496 


\section{Figure 1}

Visual description of the automated imaging setup and samples of the captured images

A) Photo of the automated imaging setup. The camera is placed on a movable platform below a transparent pane on which we placed well-plates. The height of the pane can be adjusted to accommodate different sized well plates and magnifications. Two white LED light sources provided constant light from the sides of the table. B) Example of the light conditions in the reported experiment, and an example of improved light conditions when using an electroluminescent light sheet on top of the well plates. C) Cropped images showing different copepod life stages, and which were used to measure the size of animals. Images in which the animal's outline was hard to distinguish from the background would not be used for measuring due to the bad lighting condition and removed from the analysis. 


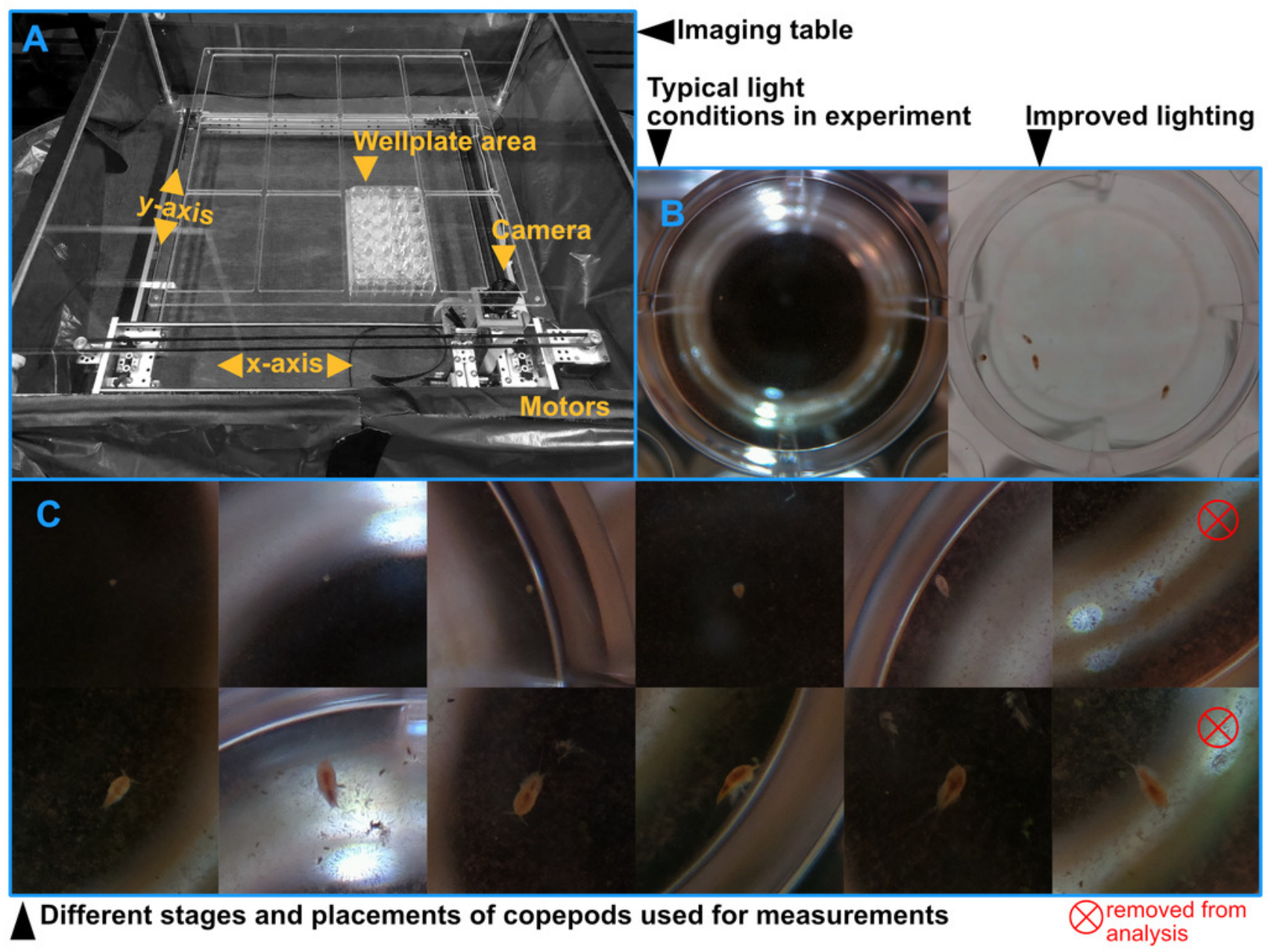


Figure 2 (on next page)

Comparison of manual and automated measurements

Comparison of copepod body lengths measured from machine images (daily average) and from daily microscope measurements acquired in Lode et al. (2018). The line represents the prediction $( \pm S E$ ) from the linear regression. 


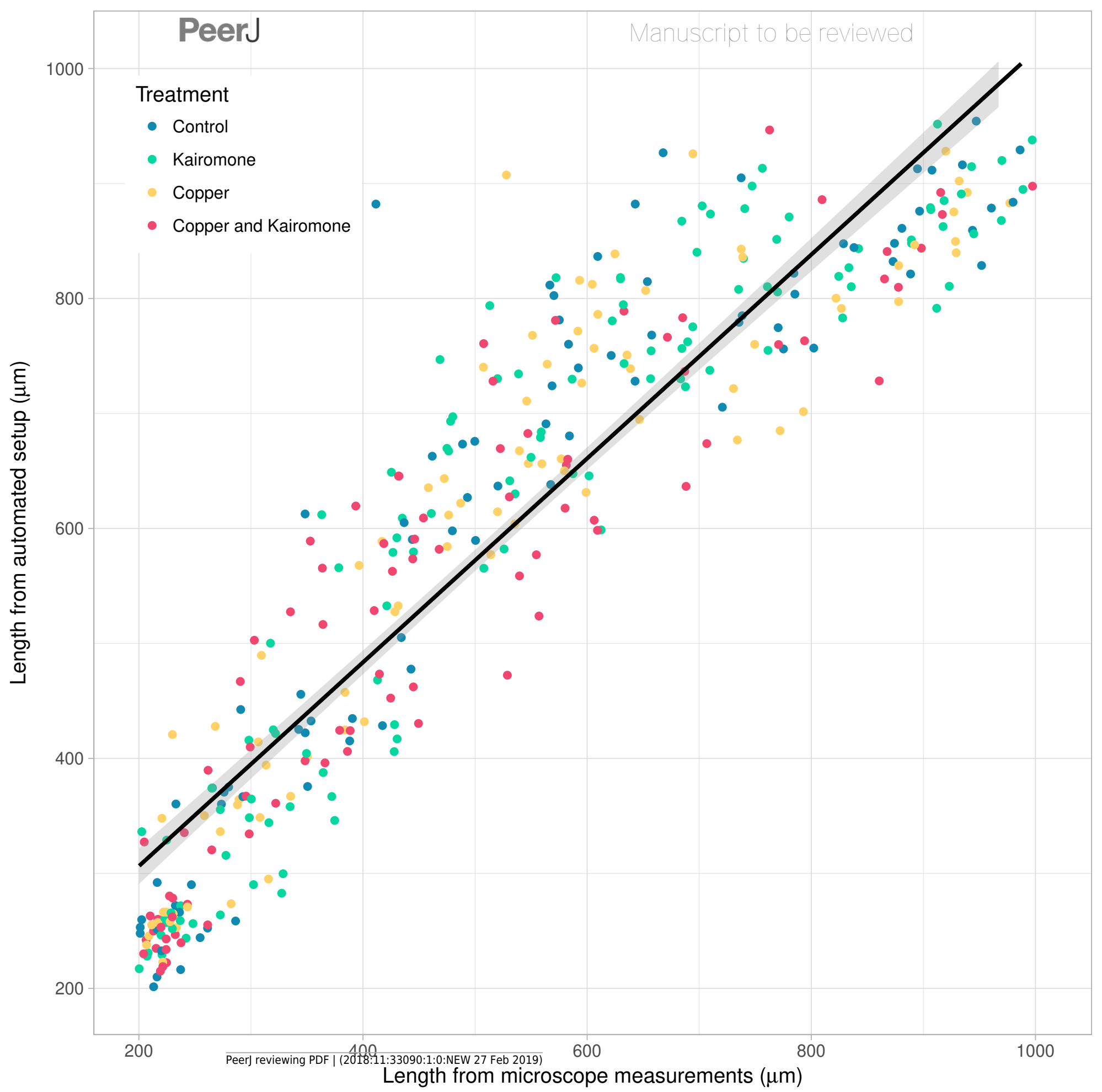




\section{Figure 3 (on next page)}

Treatment effects on the timing of $\mathrm{N} 6$ to $\mathrm{C} 1$ metamorphosis

The moment in time when nauplii $\mathrm{N} 6$ turned copepodites $\mathrm{C} 1$ depending on the copper treatment, with shapes indicating the sex as identified by the end of the experiment. Sex "Copepodites" are individuals that did not reach maturity by the end. The red dot and line indicate the mean and the SE of the raw data. 


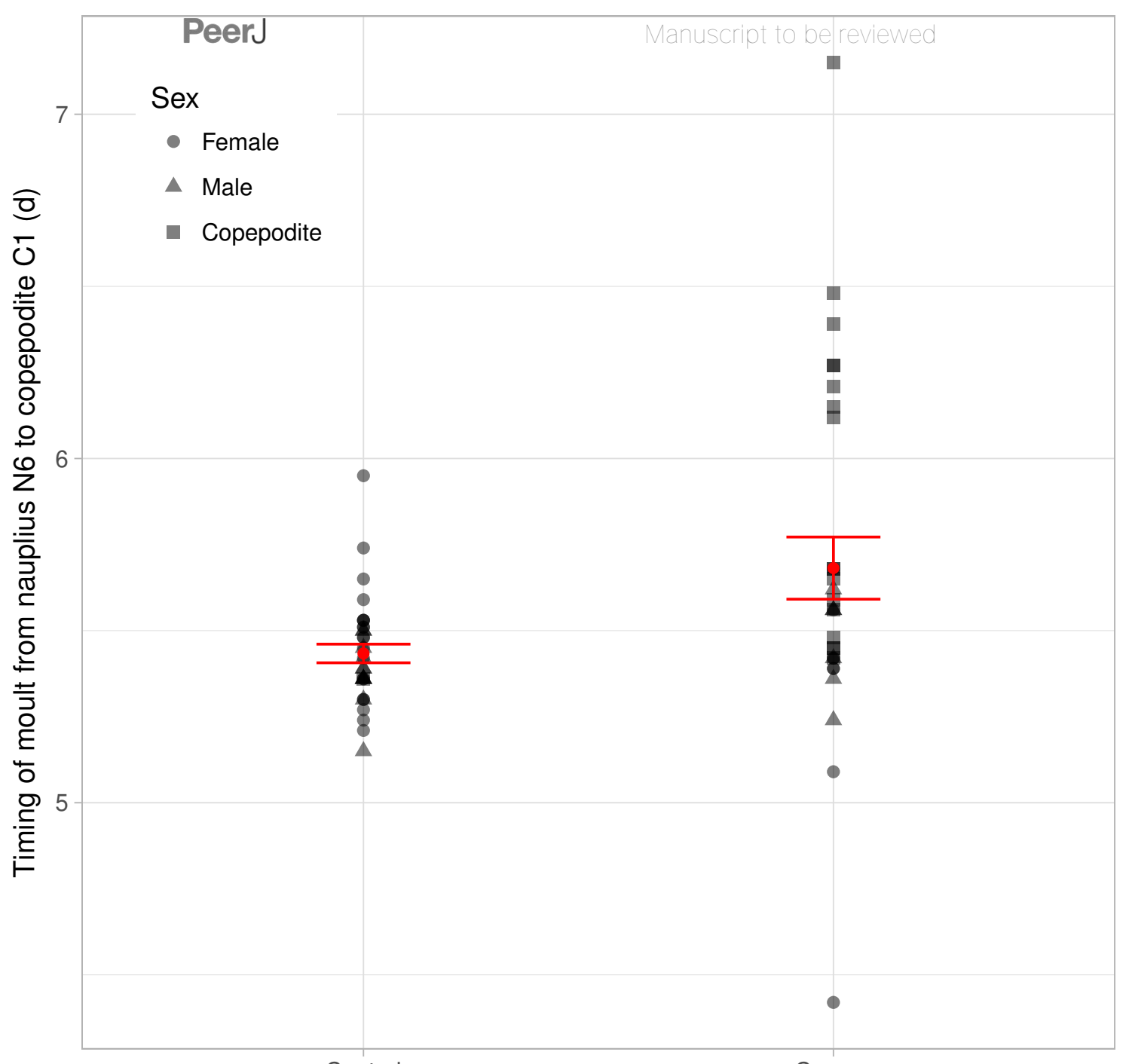


Figure 4 (on next page)

Copper and clutch ID effects on moult duration

Boxplot of the differences in N6 to $\mathrm{C} 1$ moult duration depending on clutch ID of the animals and the presence and absence of copper, the box shows median, quantiles, and the 1.5-time interquartile range is indicated by vertical lines. 


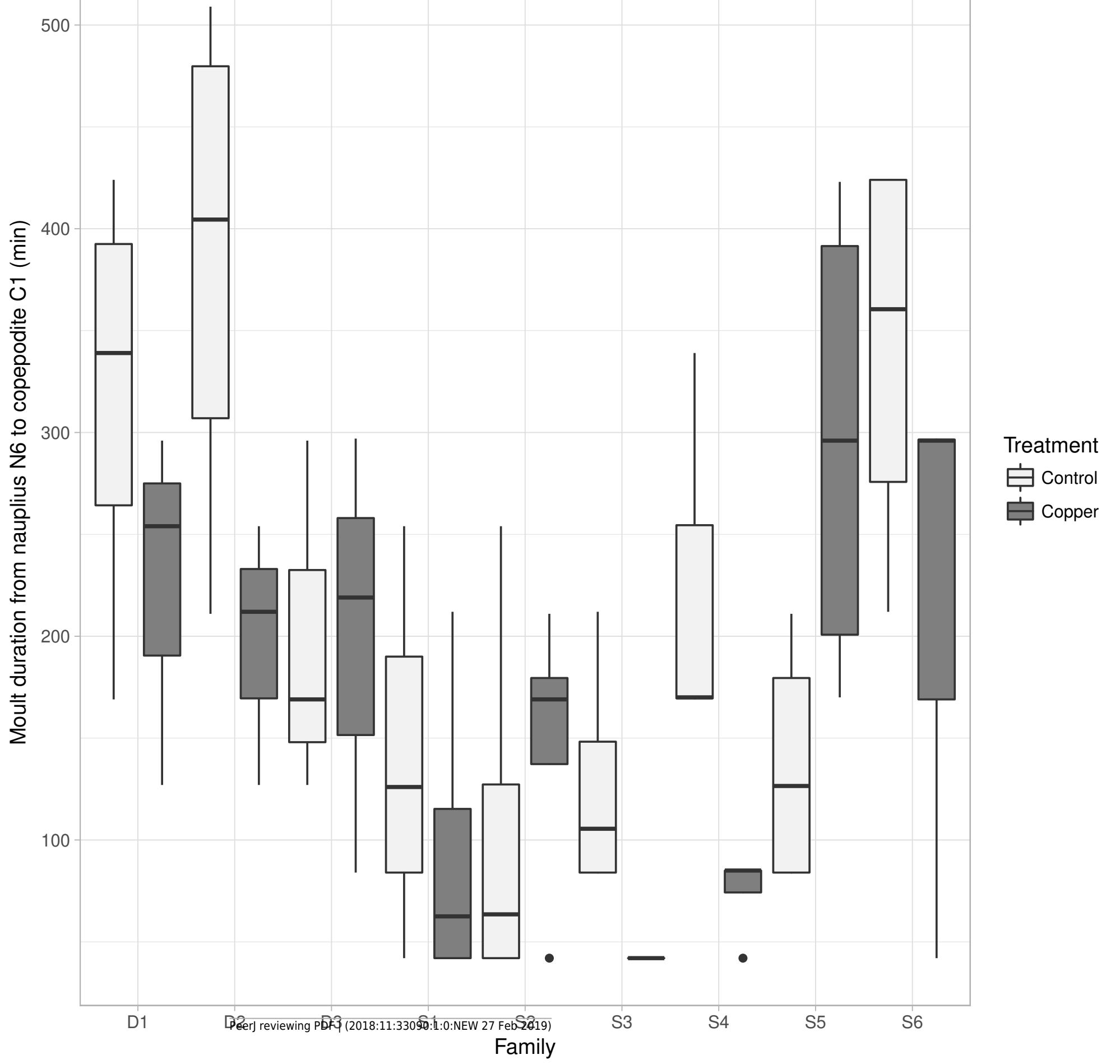


Figure $\mathbf{5}$ (on next page)

Single and interactive effects of copper and kairomones on the development of Tigriopus brevicornis

Faint lines indicate individual growth trajectories, while bold lines show the final GAM predictions averaged by treatment. While kairomones alone had little effect, the combination with copper reduced their growth in copepods more than did copper alone. 
Figure 6 (on next page)

Predicted individual growth increments as a function of treatment combination

The individual predictions are averaged over each treatment combination and time point with a 72-minute resolution. A lower average growth increment indicates a larger variability in transition timing between individuals. 
Treatment

- Control

- Kairomone

- Copper

- Copper and Kairomone

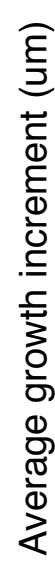

7.5

0.0

5

Peer) reviewing PDF | (2018:11:33090:1:0:NEW 27 Feb 2019) $A g e(d)$ 


\section{Table $\mathbf{1}$ (on next page)}

Model estimates of the best models testing the influence of copper, kairomone and clutch ID on the time of transition from nauplii $\mathrm{N} 6$ to copepodite $\mathrm{C} 1$ stage, as well as the duration of this major transition.

Estimates for the duration of the copepodite transition are conditional averages from model averaging of the best competing models. 


\begin{tabular}{|c|c|c|c|c|c|c|}
\hline Data & Factor & Estimate & $\begin{array}{l}\text { Std. } \\
\text { Error }\end{array}$ & & z-value & p-value \\
\hline \multicolumn{7}{|l|}{$\begin{array}{l}\text { Copepodite transition } \\
\text { time }\end{array}$} \\
\hline \multirow[t]{2}{*}{ All individuals } & (Intercept) & 1.69 & 0.03 & & 69.38 & $<0.001$ \\
\hline & Copper & 0.14 & 0.04 & & 3.95 & 0.002 \\
\hline $\begin{array}{l}\text { Duration of copepodite } \\
\text { transition }\end{array}$ & & Estimate & $\begin{array}{l}\text { Std. } \\
\text { Error }\end{array}$ & Adj. SE & $\begin{array}{l}z- \\
\text { value }\end{array}$ & $\operatorname{Pr}(>|z|)$ \\
\hline \multirow[t]{10}{*}{ All individuals } & (Intercept) & 18.05 & 2.41 & 2.51 & 7.18 & $<0.001$ \\
\hline & Copper & -2.20 & 1.24 & 1.29 & 1.70 & 0.089 \\
\hline & Clutch D2 & 1.39 & 2.96 & 3.09 & 0.45 & 0.652 \\
\hline & Clutch D3 & -4.96 & 2.92 & 3.04 & 1.63 & 0.103 \\
\hline & Clutch S1 & -7.22 & 3.00 & 3.13 & 2.31 & 0.021 \\
\hline & Clutch S2 & -8.35 & 3.03 & 3.16 & 2.64 & 0.008 \\
\hline & Clutch S3 & -9.19 & 2.96 & 3.08 & 2.99 & 0.003 \\
\hline & Clutch S4 & -4.42 & 2.92 & 3.04 & 1.46 & 0.146 \\
\hline & Clutch S5 & -2.02 & 3.13 & 3.26 & 0.62 & 0.535 \\
\hline & Clutch S6 & -1.69 & 2.89 & 3.01 & 0.56 & 0.574 \\
\hline
\end{tabular}




\section{Table 2 (on next page)}

Summary statistics of the final generalized additive model describing the influence of copper, kairomone and Clutch ID on growth over time.

The model was formulated as Length $\sim$ Clutch ID + Kairomone + Copper + te(age, by $=$

interaction(Copper, Kairomone, Clutch ID ), $k=40)+s($ age, Well, bs $=$ "fs", $m=1, k=40$ ). In the table edf represents the number of effective degrees of freedom and Ref.df the reference number of degrees of freedom used for hypothesis testing. 


\begin{tabular}{|c|c|c|c|c|}
\hline \multicolumn{5}{|l|}{ Parametric coefficients } \\
\hline & Estimate & Std. Error & t value & $\operatorname{Pr}(>|\mathbf{t}|)$ \\
\hline (Intercept (Control Clutch ID D1) & -1.20 & 0.02 & -51.09 & $<0.001$ \\
\hline Clutch ID D2 & 0.14 & 0.03 & 4.12 & $<0.001$ \\
\hline Clutch ID D3 & 0.06 & 0.04 & 1.52 & 0.129 \\
\hline Clutch ID S1 & 0.18 & 0.03 & 6.23 & $<0.001$ \\
\hline Clutch ID S2 & 0.20 & 0.03 & 6.49 & $<0.001$ \\
\hline Clutch ID S3 & 0.10 & 0.03 & 3.58 & $<0.001$ \\
\hline Clutch ID S4 & 0.16 & 0.03 & 5.41 & $<0.001$ \\
\hline Clutch ID S5 & 0.14 & 0.03 & 4.54 & $<0.001$ \\
\hline Clutch ID S6 & 0.22 & 0.03 & 6.41 & $<0.001$ \\
\hline Kairomone & -0.01 & 0.02 & -0.58 & 0.562 \\
\hline Copper & -0.11 & 0.02 & -6.58 & $<0.001$ \\
\hline \multicolumn{5}{|l|}{$\begin{array}{l}\text { Approximate significance of smooth } \\
\text { terms }\end{array}$} \\
\hline Factor combination & edf & Ref.df & F-value & P-value \\
\hline No Copper/No Kairomone/Clutch ID D1 & 1.95 & 39.00 & 2.23 & $<0.001$ \\
\hline Copper/No Kairomone/Clutch ID D1 & 0.99 & 18.00 & 4.64 & $<0.001$ \\
\hline No Copper/Kairomone/Clutch ID D1 & 0.99 & 14.00 & 5.97 & $<0.001$ \\
\hline Copper/Kairomone/Clutch ID D1 & 3.15 & 39.00 & 1.97 & $<0.001$ \\
\hline No Copper/No Kairomone/Clutch ID D2 & 5.55 & 39.00 & 2.39 & $<0.001$ \\
\hline Copper/No Kairomone/Clutch ID D2 & 3.38 & 39.00 & 2.32 & $<0.001$ \\
\hline No Copper/Kairomone/Clutch ID D2 & 3.88 & 39.00 & 3.06 & $<0.001$ \\
\hline Copper/Kairomone/Clutch ID D2 & 0.99 & 14.00 & 5.83 & $<0.001$ \\
\hline No Copper/No Kairomone/Clutch ID D3 & 2.93 & 39.00 & 2.58 & $<0.001$ \\
\hline Copper/No Kairomone/Clutch ID D3 & 4.74 & 35.00 & 2.64 & $<0.001$ \\
\hline No Copper/Kairomone/Clutch ID D3 & 2.62 & 39.00 & 2.57 & $<0.001$ \\
\hline Copper/Kairomone/Clutch ID D3 & 3.61 & 39.00 & 2.37 & $<0.001$ \\
\hline No Copper/No Kairomone/Clutch ID S1 & 1.94 & 39.00 & 1.03 & $<0.001$ \\
\hline Copper/No Kairomone/Clutch ID S1 & 0.99 & 18.00 & 4.26 & $<0.001$ \\
\hline No Copper/Kairomone/Clutch ID S1 & 2.44 & 39.00 & 2.25 & $<0.001$ \\
\hline Copper/Kairomone/Clutch ID S1 & 0.98 & 12.00 & 5.43 & $<0.001$ \\
\hline No Copper/No Kairomone/Clutch ID S2 & 2.92 & 39.00 & 2.34 & $<0.001$ \\
\hline Copper/No Kairomone/Clutch ID S2 & 2.83 & 39.00 & 2.12 & $<0.001$ \\
\hline No Copper/Kairomone/Clutch ID S2 & 3.51 & 39.00 & 2.57 & $<0.001$ \\
\hline Copper/Kairomone/Clutch ID S2 & 0.99 & 10.00 & 7.16 & $<0.001$ \\
\hline
\end{tabular}




\begin{tabular}{|l|l|l|l|l|}
\hline No Copper/No Kairomone/Clutch ID S3 & 0.99 & 13.00 & 5.80 & $<2 \mathrm{e}-16$ \\
\hline Copper/No Kairomone/Clutch ID S3 & 0.99 & 14.00 & 5.73 & $<2 \mathrm{e}-16$ \\
\hline No Copper/Kairomone/Clutch ID S3 & 1.68 & 39.00 & 2.04 & $<2 \mathrm{e}-16$ \\
\hline Copper/Kairomone/Clutch ID S3 & 2.18 & 39.00 & 1.46 & $<2 \mathrm{e}-16$ \\
\hline No Copper/No Kairomone/Clutch ID S4 & 2.43 & 39.00 & 1.10 & $<2 \mathrm{e}-16$ \\
\hline Copper/No Kairomone/Clutch ID S4 & 0.99 & 16.00 & 4.59 & $<2 \mathrm{e}-16$ \\
\hline No Copper/Kairomone/Clutch ID S4 & 2.84 & 39.00 & 2.26 & $<2 \mathrm{e}-16$ \\
\hline Copper/Kairomone/Clutch ID S4 & 0.99 & 10.00 & 7.63 & $<2 \mathrm{e}-16$ \\
\hline No Copper/No Kairomone/Clutch ID S5 & 3.15 & 39.00 & 2.71 & $<2 \mathrm{e}-16$ \\
\hline Copper/No Kairomone/Clutch ID S5 & 0.99 & 12.00 & 7.31 & $<2 \mathrm{e}-16$ \\
\hline No Copper/Kairomone/Clutch ID S5 & 3.20 & 39.00 & 2.65 & $<2 \mathrm{e}-16$ \\
\hline Copper/Kairomone/Clutch ID S5 & 2.27 & 39.00 & 2.00 & $<2 \mathrm{e}-16$ \\
\hline No Copper/No Kairomone/Clutch ID S6 & 3.80 & 39.00 & 2.77 & $<2 \mathrm{e}-16$ \\
\hline Copper/No Kairomone/Clutch ID S6 & 2.32 & 39.00 & 2.28 & $<2 \mathrm{e}-16$ \\
\hline No Copper/Kairomone/Clutch ID S6 & 3.13 & 39.00 & 2.55 & $<2 \mathrm{e}-16$ \\
\hline Copper/Kairomone/Clutch ID S6 & 0.97 & 8.00 & 4.78 & $<2 \mathrm{e}-16$ \\
\hline Well & 1312.40 & 2677.00 & 8.78 & $<2 \mathrm{e}-16$ \\
\hline
\end{tabular}




\section{Table 3 (on next page)}

Summary table for of the best general additive model describing the growth dynamics of individual copepods over age.

The model formulation was: "Growth increment $\sim$ Kairomone + Clutch ID + Copper + te(age, by $=$ interaction(Kairomone, Clutch ID, Copper), $\mathrm{k}=40$ ) $+\mathrm{s}$ (age, Well, bs = "fs", $\mathrm{m}=1, \mathrm{k}=$ 40). In the table edf represents the number of effective degrees of freedom and Ref.df the reference number of degrees of freedom used for hypothesis testing. 


\begin{tabular}{|c|c|c|c|c|}
\hline \multicolumn{5}{|l|}{ Parametric coefficients } \\
\hline & Estimate & Std. Error & t-value & $\operatorname{Pr}(>|\mathbf{t}|)$ \\
\hline (Intercept) & 0.0035 & 0.0051 & 0.70 & 0.483 \\
\hline Kairomone & 0.0043 & 0.0027 & 1.63 & 0.102 \\
\hline Clutch IDD2 & -0.0011 & 0.0057 & -0.19 & 0.851 \\
\hline Clutch IDD3 & -0.0056 & 0.0065 & -0.87 & 0.395 \\
\hline Clutch IDS1 & -0.0031 & 0.0066 & -0.48 & 0.634 \\
\hline Clutch IDS2 & -0.0072 & 0.0057 & -1.26 & 0.209 \\
\hline Clutch IDS3 & -0.0013 & 0.0057 & -0.24 & 0.814 \\
\hline Clutch IDS4 & -0.0067 & 0.0066 & -1.01 & 0.312 \\
\hline Clutch IDS5 & -0.0041 & 0.0054 & -0.77 & 0.444 \\
\hline Clutch IDS6 & 0.00074 & 0.0061 & 0.12 & 0.904 \\
\hline Copper & 0.00065 & 0.0028 & 0.23 & 0.816 \\
\hline \multicolumn{5}{|l|}{ Approximate significance of smooth terms } \\
\hline & edf & Ref.df & $\mathbf{F}$ & p-value \\
\hline NoKairomone/Clutch ID D1/NoCopper & 30.63 & 39.00 & 9.05 & $<0.001$ \\
\hline Kairomone/Clutch ID D1/NoCopper & 16.78 & 39.00 & 1.27 & $<0.001$ \\
\hline NoKairomone/Clutch ID D2/NoCopper & 27.85 & 38.00 & 7.21 & $<0.001$ \\
\hline Kairomone/Clutch ID D2/NoCopper & 32.20 & 39.00 & 15.70 & $<0.001$ \\
\hline NoKairomone/Clutch ID D3/NoCopper & 35.13 & 39.00 & 40.36 & $<0.001$ \\
\hline Kairomone/Clutch ID D3/NoCopper & 26.48 & 39.00 & 8.05 & $<0.001$ \\
\hline NoKairomone/Clutch ID S1/NoCopper & 23.27 & 39.00 & 4.88 & $<0.001$ \\
\hline Kairomone/Clutch ID S1/NoCopper & 13.07 & 38.00 & 1.75 & $<0.001$ \\
\hline NoKairomone/Clutch ID S2/NoCopper & 19.24 & 39.00 & 1.83 & $<0.001$ \\
\hline Kairomone/Clutch ID S2/NoCopper & 0.14 & 39.00 & 0.00 & 0.107 \\
\hline NoKairomone/Clutch ID S3/NoCopper & 28.26 & 38.00 & 11.56 & $<0.001$ \\
\hline Kairomone/Clutch ID S3/NoCopper & 0.00 & 39.00 & 0.00 & 0.366 \\
\hline NoKairomone/Clutch ID S4/NoCopper & 29.75 & 38.00 & 20.54 & $<0.001$ \\
\hline Kairomone/Clutch ID S4/NoCopper & 18.77 & 38.00 & 4.48 & $<0.001$ \\
\hline NoKairomone/Clutch ID S5/NoCopper & 0.00 & 39.00 & 0.00 & 0.189 \\
\hline Kairomone/Clutch ID S5/NoCopper & 0.00 & 39.00 & 0.00 & 0.199 \\
\hline NoKairomone/Clutch ID S6/NoCopper & 11.35 & 39.00 & 0.91 & $<0.001$ \\
\hline Kairomone/Clutch ID S6/NoCopper & 28.32 & 39.00 & 7.90 & $<0.001$ \\
\hline NoKairomone/Clutch ID D1/Copper & 25.90 & 39.00 & 5.83 & $<0.001$ \\
\hline Kairomone/Clutch ID D1/Copper & 8.09 & 38.00 & 0.60 & $<0.001$ \\
\hline
\end{tabular}




\begin{tabular}{|l|l|l|l|l|}
\hline NoKairomone/Clutch ID D2/Copper & 0.00 & 39.00 & 0.00 & 0.128 \\
\hline Kairomone/Clutch ID D2/Copper & 27.19 & 39.00 & 8.08 & $<0.001$ \\
\hline NoKairomone/Clutch ID D3/Copper & 0.00 & 35.00 & 0.00 & 0.540 \\
\hline Kairomone/Clutch ID D3/Copper & 25.68 & 39.00 & 8.11 & $<0.001$ \\
\hline NoKairomone/Clutch ID S1/Copper & 17.90 & 38.00 & 1.95 & $<0.001$ \\
\hline Kairomone/Clutch ID S1/Copper & 20.44 & 39.00 & 4.03 & $<0.001$ \\
\hline NoKairomone/Clutch ID S2/Copper & 11.04 & 39.00 & 0.95 & $<0.001$ \\
\hline Kairomone/Clutch ID S2/Copper & 27.59 & 39.00 & 4.62 & $<0.001$ \\
\hline NoKairomone/Clutch ID S3/Copper & 33.77 & 39.00 & 35.25 & $<0.001$ \\
\hline Kairomone/Clutch ID S3/Copper & 23.20 & 39.00 & 4.64 & $<0.001$ \\
\hline NoKairomone/Clutch ID S4/Copper & 24.30 & 39.00 & 6.16 & $<0.001$ \\
\hline Kairomone/Clutch ID S4/Copper & 29.63 & 39.00 & 15.37 & $<0.001$ \\
\hline NoKairomone/Clutch ID S5/Copper & 17.67 & 38.00 & 1.59 & $<0.001$ \\
\hline Kairomone/Clutch ID S5/Copper & 24.99 & 39.00 & 9.31 & $<0.001$ \\
\hline NoKairomone/Clutch ID S6/Copper & 1.21 & 39.00 & 0.05 & 0.029 \\
\hline Kairomone/Clutch ID S6/Copper & 11.60 & 39.00 & 0.71 & $<0.001$ \\
\hline Well & 2244.00 & 2706.00 & 322.04 & $<0.001$ \\
\hline
\end{tabular}




\section{Table 4 (on next page)}

Estimates for the final size (log transformed data) of the copepods at the end of the experiment.

Estimates are conditional model averages of the best competing models. 


\begin{tabular}{|c|c|c|c|c|c|}
\hline & Factor & Estimate & Std. Error & z-value & p-value \\
\hline \multicolumn{6}{|l|}{$\begin{array}{l}\text { Final body } \\
\text { length }\end{array}$} \\
\hline \multirow[t]{11}{*}{$\begin{array}{l}\text { All } \\
\text { individuals }\end{array}$} & $\begin{array}{l}\text { (Intercept) } \\
\text { Clutch D1 }\end{array}$ & -0.24 & 0.04 & 5.46 & $<0.001$ \\
\hline & Copper & -0.10 & 0.02 & 4.03 & $<0.001$ \\
\hline & Clutch D2 & 0.06 & 0.05 & 1.03 & 0.305 \\
\hline & Clutch D3 & 0.08 & 0.06 & 1.25 & 0.211 \\
\hline & Clutch S1 & 0.14 & 0.05 & 2.53 & 0.011 \\
\hline & Clutch S2 & 0.11 & 0.05 & 2.23 & 0.027 \\
\hline & Clutch S3 & 0.02 & 0.05 & 0.42 & 0.671 \\
\hline & Clutch S4 & 0.15 & 0.05 & 0.05 & 0.007 \\
\hline & Clutch S5 & 0.13 & 0.05 & 0.05 & 0.010 \\
\hline & Clutch S6 & 0.18 & 0.05 & 0.05 & $<0.001$ \\
\hline & Kairomone & -0.03 & 0.02 & 0.02 & 0.208 \\
\hline \multirow[t]{11}{*}{$\begin{array}{l}\text { Matured } \\
\text { individuals }\end{array}$} & $\begin{array}{l}\text { (Intercept) } \\
\text { Clutch D1 }\end{array}$ & -0.18 & 0.03 & 5.32 & $<0.001$ \\
\hline & SexM & 0.04 & 0.02 & 2.13 & 0.033 \\
\hline & Clutch D2 & 0.05 & 0.03 & 1.60 & 0.109 \\
\hline & Clutch D3 & 0.02 & 0.04 & 0.62 & 0.539 \\
\hline & Clutch S1 & 0.10 & 0.03 & 2.93 & 0.003 \\
\hline & Clutch S2 & 0.07 & 0.03 & 2.21 & 0.027 \\
\hline & Clutch S3 & 0.04 & 0.04 & 1.08 & 0.280 \\
\hline & Clutch S4 & 0.08 & 0.03 & 2.48 & 0.013 \\
\hline & Clutch S5 & 0.10 & 0.03 & 3.01 & 0.003 \\
\hline & Clutch S6 & 0.10 & 0.03 & 3.02 & 0.003 \\
\hline & Copper & 0.01 & 0.02 & 0.82 & 0.412 \\
\hline
\end{tabular}

